\title{
Influencia del programa filosofía para niños (FpN) en el desarrollo de la competencia comunicativa de los estudiantes de educación primaria
}

\section{Influence of the "Philosophy for Children" (FpN) Program in the Development of the Communicative Competence of Students in Primary Education}

\author{
Yamelis Coromoto Herrera Fuenmayor ${ }^{l}$ \\ Universidad Dr. Rafael Belloso Chacín \\ Maracaibo, Venezuela \\ yamelis10@hotmail.com \\ Eddy de Jesús Mogollón González ${ }^{2}$ \\ Universidad Dr. Rafael Belloso Chacín \\ Maracaibo, Venezuela \\ eddymogollon@gmail.com
}

Recibido 10 de noviembre de 2011 • Aceptado 23 de febrero 2012

Resumen. El objetivo de esta investigación fue desarrollar la competencia comunicativa de los estudiantes de educación primaria basado en el programa Filosofía para Niños (FpN) contextualizado. Se tomaron como referencia los aportes de Lipman (1998), Bruzual (2002), Prado (2004b), entre otros. La investigación fue explicativa, de campo, cuasiexperimental. La población fue de 255 alumnos mestizos y de la etnia Wayuu. La muestra fue de 35 niños de segundo grado (7, 8 y 9 años). El instrumento aplicado fue un test con respuestas dicotómicas. La confiabilidad se ubicó en rkk $=0,855$ (Kuder-Richardson). Se aplicó la prueba t-student como herramienta estadística. Producto de la investigación se creó un poemario denominado "Odas a tepi'chi", de lo cual se concluye que la aplicación del programa FpN fue adecuado en el desarrollo de la competencia comunicativa.

Palabras claves. Competencia comunicativa, Filosofía para niños, expresión oral y escrita, diálogo.

Abstract. The objective of this research was to develop the communicative competence of primary school students" ${ }^{3}$ based on the program "Filosofía para Niños" [Philosophy for Children] (FpN). Lipman (1998), Bruzual (2002) and Prado (2004) among other authors served as reference. Research was explanatory, field-based and quasi-experimental. The population consisted of 255 mestizo and Wayuu students. The sample selected consisted of 35 children of the second grade (7, 8 and 9 years old). The instrument applied was a test of dichotomous responses. Reliability was at rkk $=0.855$ (KuderRichardson). The t-student test was applied as a statistical tool. The book of poems "Odas a tepi 'chi" was published as a result of this study. It was concluded that the application of the FpN program was adequate in the development of the communicative competence.

Keywords. Communicative competence, Philosophy for children, oral and written expression, dialogue.

\footnotetext{
Doctorado en Ciencias de la Educación. Profesora de la Universidad Dr. Rafael Belloso Chacín, Maracaibo, Zulia. Magister Scientiarum en Literatura Venezolana . Licenciatura en Letras. Licenciada en Lengua y Literatura.

2 Doctorado en Ciencias de la Educación. Profesor Titular de la Universidad Dr. Rafael Belloso Chacín, Maracaibo, Zulia. Magister Scientiarum en Termociencias Computacional y Aplicada. Investigador de Planta del Centro de Investigación de Humanidades y Educación (CIHE/ URBE). Profesor de Postgrado de la asignatura Estadística aplicada a la investigación. Profesor de Postgrado de la asignatura Análisis e Interpretación de Reportes de Investigación.
}

3 Translator's note: Primary education in Venezuela comprises from the 1st to 6th grades. 


\section{Introducción}

Si se observa el panorama de la educación en los países latinoamericanos y, especialmente en Venezuela, se constata que se encuentra en crisis desde diferentes perspectivas: económicas, sociales, educativas, ya que la modernidad ha fracasado con su modelo tecnocrático (Carmona, 2007). La educación se ha venido desarrollando de manera racional, bancaria, deshumanizante, con el único objetivo de la rentabilidad y la competencia, por supuesto dando respuesta a la realidad que demanda el país (Ocampo, 2008).

Desde el enfoque comunicativo, la crisis es tan evidente que los estudiantes aprueban los diferentes niveles educativos: preescolar, primaria, básica media, universitaria, postgrados, con una serie de carencias en cuanto al dominio de su lengua materna; no llegan a desarrollar una competencia comunicativa eficiente. De tal manera, que los proyectos educativos de los últimos cuarenta años, que se han creado y se aplican actualmente, han sido insuficientes e inapropiados para dar respuesta a esta realidad educativa, ya que no se han generado los cambios pertinentes para resolver el problema; el cual, por el contrario pareciera incrementarse cada año (Tellez y Bruzual, 2005).

En la mayoría de los países hispanoamericanos se observa el mismo problema, de falta de competencia comunicativa en los estudiantes de todos los niveles de educación. Cabe citar los esfuerzos de Prado (2004b), con sus aportes didácticos para mejorar la expresión oral y escrita en los niños; Martínez (2001), en Colombia, con su propuesta pedagógica para leer y escribir; Accorinti (2002), en Argentina, quien desde hace más de veinte años viene promoviendo el Programa Filosofía para niños y actualmente es la presidenta del Centro de Investigación de FpN en Buenos Aires.

Sin embargo, los sistemas educativos no dan respuestas a la falta de dominio sobre la lengua materna y el problema es cada vez mayor. Los estudiantes no poseen fluidez lingüística, lo cual se demuestra en las diferentes situaciones comunicativas en que se encuentran (Castillo, 2008). De igual forma, en Venezuela el panorama no es menos grave, la falta de dominio lingüístico, la incompetencia comunicativa es muy evidente en los estudiantes de todos los subsistemas del sistema educativo.

En el estado Zulia, la realidad es más alarmante, tal vez por la gran riqueza étnica y por la presencia significativa de alumnos bilingües como es el caso de los wayuu, barí, yucpas, japreira, motilones a quienes les cuesta mucho aprender el idioma español. Lo cierto es que los estudiantes se están graduando sin adquirir una competencia comunicativa mínima; se evidencia el mal uso de la lengua española en todos los contextos comunicativos en los cuales se desenvuelven a diario (Franco,1999; 2007).

Además, la inclinación por el estudio de las competencia comunicativa no es reciente; diferentes investigaciones han arrojado aportes significativos, como en España las de Prado (2004a); en Colombia, los aportes de Martínez (2001), y Bruzual (2002), Molero (1998) y Franco (2007), en el Estado Zulia, han determinado fallas graves en la enseñanza de la lengua materna y, al mismo tiempo, para resolver el problema han presentado propuestas de importancia, pero sin apoyo pragmático.

Para mejorar la problemática anteriormente planteada, es necesario que los docentes planifiquen estrategias adecuadas para el desarrollo de la competencia comunicativa desde muy temprana edad, y así elevar el rendimiento académico de los alumnos. Por ello, resulta pertinente el enfoque que brinda el Programa Filosofía para niños de Lipman (1998), el cual conlleva a desarrollar una competencia cognitiva; este autor hace referencia a la apropiación y construcción del saber a partir de la aplicación del diálogo como motor del pensamiento crítico reflexivo; fundamentándose en teorías de la historia epistemológica de las diferentes disciplinas. 
Por tal motivo, el objetivo de este trabajo de investigación es determinar el efecto del Programa Filosofía para niños en el desarrollo de la competencia comunicativa de los estudiantes de educación primaria en la U.E "María Luisa Niño” ubicada en el Municipio Mara.

\section{Desarrollo teórico}

\section{Competencia comunicativa}

Según expone Bruzual (2002), fue Chomsky quien, con sus trabajos realizados $(1965,1969$ y 1983), introdujo el concepto de competencia en la Lingüística. La define como el conocimiento de la gramática; de las reglas lingüísticas necesarias para generar frases gramaticalmente correctas y, al mismo tiempo, la diferencia de actuación explicando que es el uso concreto de la lengua. Tomando como referencia los aportes de Hymes (1971), esta autora explica que este último autor es quien aporta el concepto de competencia comunicativa para ampliar el concepto de competencia lingüística chomskyano y le da una orientación más funcional y contextual. Para Bruzual (2002, p. 24), la competencia comunicativa va más allá del dominio lingüístico, siendo esta la capacidad de los individuos para saber qué, a quien, cuándo, y cómo expresarse: “(...) es el conjunto de procesos que el hablante/escritor debe utilizar para producir y comprender distintos tipos de discursos adecuados a la situación y al contexto de comunicación y al grado de formalización requerido (...)"; es decir, la competencia comunicativa implica el conocimiento y dominio de normas, estrategias para la producción de mensajes acordes con la intención y situación en que se encuentre el individuo.

Por otro lado, Bruzual (2002) plantea que el enfoque comunicativo busca desarrollar la competencia comunicativa. Por ello, se apega a la clasificación que hacen Lomas (1999), Canale y Swain (1980), quienes afirman que la competencia comunicativa está integrada por las subcompetencias: lingüística, sociolingüística, discursiva o textual y estratégica. Más adelante se suma la competencia pragmática propuesta por Serrón (2002).

De igual manera, Prado (2004b) parte de las ideas expuestas por Hymes (1971), y más tarde concretadas por Canale (1983), quienes plantean que la competencia comunicativa está integrada por una serie de subcompetencias:

a) Competencia gramatical: es la que se adquiere a través del dominio del código lingüístico, verbal y no verbal, conocimiento de las reglas ortográficas, lexicales, semánticas, fonológicas, morfológicas.

b) Competencia sociolingüística: implica adecuar el mensaje a los diversos contextos, atendiendo normas de interacción, situación, intenciones y convenciones sociales.

c) Competencia discursiva: se refiere al dominio de las normas gramaticales, sintácticas y semánticas para producir textos orales o escritos en diferentes géneros; implica cohesión textual y coherencia del discurso.

d) Competencia estratégica: definida como aquella que consiste en la habilidad de aplicar la comunicación verbal y no verbal de manera estratégica con efectividad. 
Asimismo, es importante señalar los aportes de Saussure (1983, p. 59) en cuanto a la competencia lingüística; la cual “(...) se ocupa de cualquier manifestación, vigente o pasada, del lenguaje humano, esto es, se ocupa del estudio de la lengua hablada en cualquier pueblo, independientemente de su grado de civilización, se trate de pueblos con una cultura antigua o contemporánea. Igualmente estudia tanto la forma de expresiones correctas o bien habladas, como las no correctas desde el punto de vista de la gramática".

El nivel fonético y fonológico: Saussure (1983) comienza por especificar que la fonética se ocupa de estudiar los sonidos de una lengua, cómo se combinan, cómo hace el hablante para producir los distintos sonidos al emitirlos y cómo viajan a través del aire para que finalmente lleguen al receptor. De igual manera explica que la fonología se encarga de formalizar los datos sobre los diferentes tipos de sonidos que posee una lengua para poder determinar las funciones distintivas que ejercen en dicha lengua; es decir, estudia sus elementos fónicos específicamente el valor distintivo y funcional.

El nivel morfológico y sintáctico: Para Saussure (1983) la morfología estudia la estructura de las palabras, su formación, sus alteraciones, sus flexiones para indicar los diferentes accidentes gramaticales: la conjugación, género, número, la derivación y las clases de palabras, sus funciones dentro de los contextos oracionales. Este autor también define la sintaxis como la disciplina que se encarga del estudio de las relaciones de las palabras en las oraciones, su orden lógico según la jerarquización de los elementos en la línea del mensaje.

Para Saussure (1983), el léxico está constituido por las unidades léxicas o lexemas que conforman la lengua y la semántica las define como la ciencia del sentido, se encarga de estudiar los significados de las palabras, el contenido del mensaje y sus combinaciones según el contexto oracional; examina el significado de cada palabra y de los enunciados para utilizarlos con propiedad.

De igual manera, el mencionado autor define el nivel pragmático como la capacidad para lograr los propósitos de comunicación en una lengua: para ordenar, asustar, especular, rechazar, retractarse, negar, clasificar, preguntar, perdonar, felicitar, saludar y agradecer, entre otros. Los actos de habla pueden variar de una cultura a otra, ya que son el reflejo de diferentes sistemas de valores.

Competencia sociolinguiística o etnografía del habla, como la denominó Hymes (1996), es una descripción en términos culturales de los usos pautados de la lengua y el habla, o sea, las reglas de interacción social o de un grupo, institución, comunidad o sociedad particular. Esta definición hace que se deban tomar en cuenta los siguientes aspectos para entenderlos:

- Los recursos sociolingüísticos de una comunidad en particular, incluyendo los no gramaticales y potenciales lingüísticos. Las interacciones y organizaciones pautadas de los diversos tipos de discurso e interacción social en la comunidad.

- Las relaciones de dichas pautas se hablan con otros aspectos de la cultura de la comunidad tales como: organización social, religión, política, etc.

- El uso y explotación de los recursos en el discurso: situación del habla, evento de habla y acto de habla. Dentro de una comunidad lingüística se pueden detectar muchas situaciones de habla que se asocian con el uso del lenguaje o que están marcadas por su ausencia, como ejemplo: fiestas, comidas, clases, ceremonias. Un evento del habla, según Hymes (1994), es la actividad o aspectos de la misma que está regido por reglas o normas para el uso lingüístico, por ejemplo, una conversación privada, una discusión o una prédica. 
- La sociolingüística está integrada por el modelo de Speaking, la competencia interaccional y las reglas de interacción social. A continuación se explican brevemente:

Modelo de Speaking: La competencia sociolingüística implica unas categorías de análisis según Hymes (1996), las cuales conforman el siguiente modelo; este autor pone de relieve el concepto de la situación social a través del modelo Speaking, donde cada letra representa un concepto de análisis en la situación comunicativa.

Competencia interaccional: Involucra el conocimiento y el uso de reglas no escritas de interacción de diversas situaciones de comunicación en una comunidad sociocultural-lingüística dada. Incluye, entre otras cosas, saber cómo iniciar, continuar y manejar las conversaciones y negociar el significado con otras personas; el tipo de lenguaje corporal apropiado; el contacto visual y la proximidad entre los hablantes y el actuar en consonancia con esas reglas.

\section{Competencia discursiva o textual}

Según Lomas (1999), citado por Bruzual (2002), la competencia discursiva es la relativa a los conocimientos y habilidades para comprender, producir e interpretar diferentes tipos de discursos e interpretar y producir textos coherentes, fluidos, aplicando formas gramaticales con cohesión textual, atendiendo el dinamismo, la interacción comunicativa, el uso del lenguaje contextos particulares. No solo es importante tener dominio del sistema lingüístico, de la sociolingüística, sino también poseer competencia para la producción de discursos orales y escritos como respuesta ante las diversas situaciones de comunicación que enfrenta el hablante a diario.

- La cohesión discursiva: Uno de los elementos más importantes en el discurso es la cohesión; Martínez (2001, p. 41), dice que “(...) se refiere a la manera como los usuarios de una lengua organizan un desarrollo proposicional en el texto oral o escrito (...) La cohesión indica la manera como la secuencia de unidades de información se provee de unidad conceptual relacional a través de unidades lingüísticas de tipo léxico o gramatical".

- La coherencia discursiva: Es importante referir que la coherencia discursiva también es un elemento esencial en el discurso. Según Martínez (2001, p. 107), “(...) se refiere a la manera como las proposiciones son utilizadas por los usuarios de una lengua para realizar actos, para, además de decir algo hacer algo con lo que se dice: hacer una invitación, una promesa, una repetición, dar un consejo, hacer una advertencia, una afirmación, una proposición”.

\section{Competencia estratégica}

Según Lomas (1999), citado por Bruzual (2002, p. 28), es la competencia “(...) que se refiere al dominio de los recursos que pueden ser utilizados para resolver los diversos problemas que se producen en el intercambio comunicativo (...)". Se adquiere a través de las estrategias de comunicación tanto verbal como no verbal que hay que poner en acción con vistas a compensar rupturas en la comunicación y mejorar la efectividad debido a la insuficiencias en una o varias competencias. Es muy importante para el aprendizaje de una segunda lengua. 
Esta investigación también se fundamente teóricamente en los aportes de Matthew Lipman con su Programa Filosofía para Niños $(\mathrm{FpN})$. Los investigadores toman como referencia el método del "diálogo filosófico" y "comunidad de investigación", pero con un enfoque lingüístico con el objetivo de demostrar que los niños también pueden alcanzar competencias comunicativas a partir de estas prácticas en el aula, sin alejarse del propósito principal del Programa que es el desarrollo del pensamiento crítico-reflexivo en los niños desde muy temprana edad (Lipman, 1998); ya que el lenguaje y pensamiento se desarrollan de manera paralela.

\section{Currículo del programa de Filosofía para niños y niñas}

El autor Lipman (1998) ha hecho aportes muy significativos para el desarrollo del pensamiento, la creatividad y la reflexión en los niños desde muy temprana edad, a través de la creación de un material concreto, bien estructurado y acorde a la edad. Este material consta de siete novelas y sus correspondientes manuales de apoyo, donde los protagonistas son los niños y su vida se desarrolla en situaciones y circunstancias cotidianas con las cuales se identifican como lectores.

El manual que acompaña a cada novela permite orientar al docente para desarrollar el proceso en virtud de la investigación filosófica con sus alumnos, también contiene una explicación de los distintos temas y conceptos filosóficos que aparecen en la novela, con la finalidad de generar debates y discusiones en el aula; luego se presentan ejercicios y problemas que permiten la libertad comunicacional de los niños y reflexiones a partir de ello.

El objetivo fundamental del planteamiento de Lipman es convertir el aula tradicional en una comunidad de diálogo o de investigación conjunta, participativa y cooperativa, en la que alumnos y profesores buscan las respuestas a las cuestiones planteadas a través de un trabajo colaborativo.

\section{Objetivos del programa de Filosofía para niños y niñas} siguientes:

Entre los objetivos que persigue el programa de Filosofía para niños se encuentran los

- Propiciar la actitud reflexiva, crítica y creativa del niño, para lograr que los mismos sean personas autónomas, mejoren su rendimiento académico, a la vez que sus actitudes de convivencia con los demás.

- Desarrollar destrezas de razonamiento, facilitándoles a los niños, niñas y adolescentes un ámbito para la expresión libre de sus puntos de vista y ayudándolos a encontrar significado en los objetos de conocimiento, así como al planteamiento y replanteamiento de nuevas teorías de significados a partir de las experiencias obtenidas.

- Familiarizar a los niños con la reflexión filosófica, entendida de modo amplio y como análisis crítico y discusión de sus experiencias e intereses, brindar importancia al trabajo con los materiales adecuados que propicien en el niño la reflexión y el desarrollo del pensamiento en forma lógica y coherente.

- Reforzar los aspectos emocionales y cognitivos de la experiencia educativa con base en la experiencia propia. Es importante sensibilizar al niño hacia las necesidades y problemas de los 
demás seres humanos, más allá del aula y de las disciplinas, ydotarlos de armas para enfrentar el adoctrinamiento.

- Crear un ambiente favorable para el aprendizaje compartido, convirtiendo el aula en una comunidad de trabajo creativo e investigación, autocrítica y autorreflexión

- Destacar el papel del diálogo como portador de valores y como vehículo de reflexión en común, el cual contribuye al enriquecimiento personal y a la asunción de posiciones críticas ante la vida (Lipman, 1998).

\section{EL currículo de Lipman}

El currículo creado por Lipman (2002), entre 1969 y 1980, está formado por las siguientes siete novelas por edades, en sus títulos originales: Elfie, Kio y Agus, Pixie, El descubrimiento de Harry Stottlemeier, Lisa, Suki y Mark. Se describe cada una brevemente:

1. Elfie (4 a 6 años): Se centra en el conocimiento de uno mismo y en la exploración de los aspectos problemáticos de la experiencia.

2. Gus y Pixie (8 a 9 años): Permite desarrollar la capacidad de asombro de los niños ante las maravillas presentes en el mundo que les rodea.

3. El descubrimiento de Harry (10 años): Ofrece un modelo de diálogo para que los niños apliquen la lógica del lenguaje en todas las situaciones comunicativas que se les presenten en su entorno sociocultural; y de esta manera puedan interactuar a través del lenguaje

4. Pixi (11 años): Ayuda a desarrollar el conocimiento científico que se fundamenta con la objetividad, su finalidad es que el niño sea más específico para investigar y para las innovaciones científicas.

5. Lisa (12 años): Hace énfasis en los valores que le van a permitir al niño ser un buen ciudadano y poder insertarse con éxito en la sociedad con sentido de humildad y humanidad.

6. Suki (13 años): Ayuda a dominar la escritura a través del estudio gramatical, elemento indisoluble de la lengua y medio de expresión universal.

7. Mark (14 a 16 Años): Presenta la importancia de analizar cómo el ser humano es la esencia de toda sociedad, a través de las discusiones de diferentes enfoques.

\section{Marco metodológico}

Se realizó un estudio de campo, explicativo, con un diseño cuasiexperimental y longitudinal. La población estuvo conformada por 255 alumnos de educación básica, de la unidad educativa "María Luisa Niño", ubicada en sector Dos Bocas (zona rural), parroquia Luis de Vicente, municipio Mara, Edo Zulia. La muestra se asumió como muestreo no probabilístico intencional u opinático (grupos intactos) (Arias, 2006) y estuvo conformada por 35 niños de segundo grado (7, 8 y 9 años). EL grupo de control estuvo conformado por 17 niños (11 de sexo masculino y 6, femenino) y el grupo experimental por 18 niños (12 de sexo masculino y 6 , femenino)

Se seleccionaron los grupos considerando que son estudiantes que disponen de conocimientos previos de lectura, se encuentran ubicados de acuerdo con sus edades y sexo; por consiguiente, son grupos homogéneos. 


\section{URL: http://www.una.ac.cr/educare}

Para evaluar las competencias comunicativas de los alumnos se aplicó un test denominado: Cuestionario tipo lista de cotejo dirigido a los docentes de 2do grado de la U.E "María Luisa Niño, estructurado en cuatro partes, donde cada parte es una dimensión con sus indicadores (14 en total); con respuestas dicotómicas con valoración de un punto para cada respuesta del Sí (Sí: sí posee competencia; No: no posee competencia). La puntuación total de la prueba fue de 40 puntos. La aplicación del test se realizó en presencia de los investigadores, para evitar sesgos y mantener una mayor uniformidad de criterios. Las competencias evaluadas se muestran en la tabla 1.

Tabla 1

Competencias comunicativas

\begin{tabular}{|l|l|}
\hline \multicolumn{2}{|c|}{ SUBCOMPETENCIAS } \\
\hline Tipo de subcompetencia & Niveles \\
\hline Gramatical & Ortográfico \\
\hline Lingüística & Fonético y fonológico, morfológico y sintáctico, léxico y semántico, pragmático \\
\hline Sociolingüística & $\begin{array}{l}\text { Reglas de interacción social, modelo de Speaking, } \\
\text { competencia interaccional, competencia cultural }\end{array}$ \\
\hline Discursiva & El discurso, cohesión, coherencia \\
\hline Estratégica & Comunicación verbal, comunicación no verbal \\
\hline
\end{tabular}

Nota. Elaboración propia de los autores (2011)

\section{Validez y confiabilidad del instrumento}

La confiabilidad de la prueba se midió a través de una prueba piloto con aplicación test-retest, utilizando la fórmula de Kuder-Richardson $\left(\mathrm{r}_{\mathrm{kk}}\right.$ ) Chávez (2007). La confiabilidad del cuestionario dio como resultado un coeficiente de $r_{k \mathrm{k}}=0,855$, que indicó la consistencia en las respuestas. En esta investigación se aplicaron los tratamientos estadísticos como: $t$ student al pretest y al postest para la comparación de medias y valoración de la estrategia. Para los cálculos estadísticos se utilizó el paquete informático SPSS-12.

\section{Procedimiento de la investigación}

1. Se preparó un protocolo de sensibilización con los padres, representantes y maestros que permitió desarrollar y sensibilizar la población en estudio, para la aplicación del programa de Filosofía para niños a partir del 09 de mayo de 2011.

2. Se implementó la metodología del Programa Filosofía para Niños basado en el diálogo filosófico para alcanzar competencias comunicativas orales y escritas con el grupo experimental (GE), desde el 09 de mayo al 26 de junio con un plan de acción previamente elaborado por los investigadores, por sesiones. 


\section{Análisis y discusión de los resultados}

A continuación se analizan los resultados por los niveles, dimensiones y variables de estudio. En la tabla 2 se describen los resultados, (en porcentajes) del pretest y postest.

Tabla 2

Resultados del pre y postest

\begin{tabular}{|c|c|c|c|c|c|c|c|c|}
\hline \multicolumn{9}{|c|}{ COMPETENCIAS COMUNICATIVAS } \\
\hline & \multicolumn{4}{|c|}{ PRE-TEST } & \multicolumn{4}{|c|}{ POST-TEST } \\
\hline \multirow{2}{*}{$\begin{array}{l}\text { Tipo de } \\
\text { competencia }\end{array}$} & \multicolumn{2}{|c|}{$\begin{array}{c}\text { Grupo de control } \\
(\%)\end{array}$} & \multicolumn{2}{|c|}{$\begin{array}{c}\text { Grupo experimental } \\
(\%)\end{array}$} & \multicolumn{2}{|c|}{$\begin{array}{c}\text { Grupo de control } \\
(\%)\end{array}$} & \multicolumn{2}{|c|}{$\begin{array}{c}\text { Grupo experimental } \\
(\%)\end{array}$} \\
\hline & SI & $\mathrm{NO}$ & SI & NO & SI & NO & SI & $\mathrm{NO}$ \\
\hline Gramatical & 07,82 & 92,16 & 11,11 & 88,89 & 10,14 & 89,86 & 79,63 & 20,37 \\
\hline Lingüística & 26,47 & 73,53 & 25,93 & 74,07 & 29,41 & 70,59 & 68,05 & 31,95 \\
\hline Sociolingüística & 32,09 & 67,91 & 30,30 & 69,70 & 33,69 & 66,31 & 67,17 & 32.82 \\
\hline Discursiva & 16,99 & 83,01 & 08,64 & 91,36 & 17,65 & 83,35 & 64,32 & 35,68 \\
\hline Estratégica & 16,47 & 83,53 & 29,00 & 80,00 & 22,35 & 77,65 & 71,11 & 28,89 \\
\hline
\end{tabular}

Nota. Elaboración propia de los autores (2011)

En este aspecto, se determinó que el grupo de niños analizados, en el GC y GE para el pretest, no poseen competencia comunicativa. En la competencia gramatical se muestra un porcentaje de $92,16 \%$ de respuestas con el No (No posee competencia) en el GC; y 88,89\% en el GE. En la competencia lingüística se presentó un alto porcentaje de No posee competencia $(73,53 \%$ para el GC y 74,07 para el GE). En cuanto a la competencia sociolingüística, se evidencia un alto porcentaje de No (67,91 y 69,70 respectivamente), lo cual indica que no poseen la capacidad para interactuar con los demás por medio de su lengua. En relación con la competencia discursiva, en el pretest se presentó un alto porcentaje de No $(83,01 \mathrm{GC}$ y 91,36 GE), lo que determina la falta de capacidad para producir discursos, tanto orales como escritos, con cohesión y coherencia, y con adecuación al contexto comunicativo en que se encuentren. En relación con la competencia estratégica se presentó un alto porcentaje del No (83,53 GC y $80 \mathrm{GE})$, lo que determina la falta de capacidad de los niños para comunicarse de manera verbal (oral-escrita) y no verbal (códigos para y extralingüísticos).

De las evidencias anteriores, se deduce que los resultados del pretest se consideran negativos tanto para el grupo control como el grupo experimental. Los resultados del pretest mostraron que los niños son incapaces de relacionar oraciones utilizando bien los conectores, no ordenaron correctamente las ideas al expresarlas, ni estas fueron claras ni adecuadas al tema de conversación. Por lo tanto, se demuestra que no poseen competencia discursiva al comparar los resultados con la teoría de Bruzual (2002), quien explica que la competencia discursiva es la habilidad de producir e interpretar diferentes tipos de discursos e interpretar y producir textos coherentes, fluidos, con aplicación de formas gramaticales con cohesión textual; es el uso del lenguaje en contextos particulares. 


\section{URL: http://www.una.ac.cr/educare}

Los resultados del postest también se muestran en la tabla 2. Como se puede observar, luego de aplicada la metodología del Programa Filosofía para Niños, muestran una mejora considerable los sujetos del Grupo Experimental (GE).

En la tabla 2 y 3 se describen los promedios obtenidos por ambos grupos durante la aplicación del pre-test, se observa que ambos grupos presentan promedios muy bajos, con respecto a la puntuación total de 40 puntos. El resultado evidente da como punto significativo que ambos grupos no poseen competencias comunicativas al iniciar la experiencia.

Tabla 3

Puntuaciones obtenidas del pretest (ambos grupos)

\begin{tabular}{|l|c|c|}
\hline \multicolumn{3}{|c|}{$\begin{array}{c}\text { Resultados preprueba } \\
\text { Puntuación } \\
\text { Máxima: 40 puntos }\end{array}$} \\
\hline & Grupo control & Grupo experimental \\
\hline Promedio (puntos) & 9,2941 & 8,7647 \\
\hline Desviación estándar & 6,58374 & 5,22649 \\
\hline Mínimo & 1,00 & 3,00 \\
\hline Máximo & 24,00 & 20,00 \\
\hline
\end{tabular}

Nota. SPSS versión 12

Tabla 4

Puntuaciones obtenidas del postest (ambos grupos)

\begin{tabular}{|l|c|c|c|}
\hline \multicolumn{4}{|c|}{$\begin{array}{c}\text { Resultados Posprueba } \\
\text { Puntuación } \\
\text { Máxima: 40 puntos }\end{array}$} \\
\hline & \multicolumn{3}{|c|}{ Promedio para ambos grupos Postest } \\
\hline & Promedio (puntos) & $\mathrm{n}$ & Desviación estándar \\
\hline Control & 11,2353 & 17 & 7,35297 \\
\hline Experimental & 26,3889 & 18 & 8,97801 \\
\hline
\end{tabular}

Nota. SPSS versión 12

Según los resultados de la tabla anterior el promedio de ambos grupos cambió de manera significativa. Se observa que el promedio del grupo de control (GC) alcanzó un puntaje de 11,23. En relación con el grupo experimental (GE), el promedio fue de 26,38. La desviación estándar del GC es 7,35 y GE es de 8,9. Es importante aquí resaltar que el promedio del grupo experimental es mucho más elevado que el del grupo de control. Se puede observar que ambos grupos han mejorado su puntaje, pero el programa de FpN fue ventajoso para el grupo experimental. 


\section{PRUEBA DE $\mathrm{t}$ - Student (PRETEST)}

\section{Pruebas de muestra independientes}

Como $\mathrm{N}<100$ ambas muestras, es necesario comprobar normalidad en ambas aplicando la prueba Test de Kolmogorov-Smirnov.

Para ambas muestras deben cumplirse:

Ho = Los datos (puntuaciones del grupo control- PRETEST) provienen de una población normal.

H1 = Los datos (puntuaciones del grupo de control- PRETEST) no provienen de una población normal.

Según el resultado arrojado por el SPSS, versión 12, para el test de Kolmogorov-Smirnov en ambos grupos se tomó la siguiente decisión: Como $\mathrm{p}=0,763>0,05$ se acepta Ho (grupo de control). Como $\mathrm{p}=0,324>0,05$ se acepta Ho (grupo experimental), hay normalidad en ambas muestras.

La prueba homocedasticidad (igualdad de varianzas)

Aplicando Test de Levene. Se asumen las siguientes hipótesis:

Ho = Las varianzas de ambos grupos no son diferentes

$\mathrm{Ha}=$ Las varianzas de ambos grupos son diferentes

Los resultados de la prueba Levene:

Como $\mathrm{p}=0,175>0,05$ se acepta Ho, existe homocedasticidad en ambas muestras. Por lo tanto, es aplicable la prueba $\mathrm{t}$ - student. (Prueba si las muestras son independientes en el pre- test). Se consideran las hipótesis:

Ho $=$ No existen diferencias entre las medias de los dos grupos

$\mathrm{Ha}=$ Existen diferencias entre las medias de los dos grupos

Como $\mathrm{p}=0,715>0,05$ se acepta Ho.

Estas pruebas nos indican que las muestras son homogéneas e independientes en el pretest (Chávez, 2007).

\section{Aplicación de la prueba $\mathbf{t}$ - Student para comparar las medias de ambos grupos (Postest)}

Planteamiento de la hipótesis. 


\section{URL: http://www.una.ac.cr/educare}

Hipótesis alterna (Ha): $\mu_{1 \neq} \mu_{2}$ La media de ambos grupos son diferentes Hipótesis nula (Ho): $\mu_{1=} \mu_{2}$ La media de ambos grupos son iguales.

\section{Probando normalidad y homocedasticidad}

Prueba de normalidad (test de Kolmogorov-Smirnov)

Como $\mathrm{p}=0,763>0,05$ se acepta Ho (grupo de control).

Como $\mathrm{p}=0,227>0,05$ se acepta Ho (grupo experimental)

Por los resultados arrojados se determinó que hay normalidad en ambas muestras.

Prueba de normalidad (Test de Levene): Como $\mathrm{p}=0,970>0,05$ se acepta Ho, existe homocedasticidad en ambas muestra (igualdad de varianzas).

Tabla 5

Prueba $t$-Student

\begin{tabular}{|c|l|c|l|c|c|c|}
\hline $\mathrm{T}$ & Prueba del Postest & \multicolumn{5}{|c|}{ Independent Samples Test } \\
\hline & $\mathrm{t}-$ student & $\mathrm{t}$ & $\mathrm{df}$ & Sig. (2-tailed) & Mean Difference & Std. Error Difference \\
\hline & $\begin{array}{l}\text { Equal variances } \\
\text { assumed }\end{array}$ & $-5,444$ & 33 &, 000 & $-15,15359$ & 2,78348 \\
\hline & $\begin{array}{l}\text { Equal variances } \\
\text { not assumed }\end{array}$ & $-5,476$ & 32,373 &, 000 & $-15,15359$ & 2,76738 \\
\hline
\end{tabular}

Fuente: SPSS versión 12

Los resultados en la tabla 5 muestran una diferencia en ambos grupos $\mathrm{p}=0,000$ (igual variancias asumidas), siendo $\mathrm{p}<0,005)$. Por lo tanto, se rechaza la hipótesis nula Ho y se acepta la Ha de que el grupo experimental reporta una mejora bastante significativa de acuerdo con la metodología del Programa de FpN aplicado.

\section{Propuesta de la investigación}

Por razones de espacio, solo se muestran las fases relevantes de la propuesta. Los autores pueden enviar la misma a todos aquellos lectores interesados, siempre que se reconozcan los derechos de autoría y se utilice con fines académicos.

Se propone la inserción del Programa de Filosofía para niños contextualizado en el currículo del nivel de educación primaria de educación básica, por medio de la capacitación de los docentes, para ofrecer una educación de calidad con la estimulación de la lectura crítica reflexiva, mediante la aplicación del método dialógico propuesto por Lipman (1998). 
Dicha propuesta tiene, por un lado, la finalidad de darles alas a las mentes infantiles; es decir, desarrollar el pensamiento crítico-reflexivo en los niños desde temprana edad; así como también que, por el disfrute de la lectura, sean capaces de indagar, reflexionar, inferir, ser críticos, sacar conclusiones, hacer predicciones, dar opiniones, hacer comparaciones y, sobre todo, logren expresarse con fluidez y propiedad libremente. Por otro lado, busca sensibilizar al docente para que aplique el diálogo como estrategia de aprendizaje.

La propuesta está estructurada en dos partes. La primera incluye la descripción del plan de acción que se diseñó para aplicar el programa FpN con los estudiantes del 2do grado de educación primaria en la U.E "María Luisa Niño": metodología, actividades, recursos y descripción de los hallazgos. Se trabajó por sesiones con diferentes lecturas: Elfie, con su respectivo manual (Lipman y Gazard, 2001), luego se seleccionaron otras lecturas debido a que los niños no se sentían motivados ni identificados con la historia ni con los nombres de los personajes de Lipman por ser extranjeros (Elfie, Seth, Bruce, Sra. Tripp, Sr. Sprockett). Estas son: El principito, de De Saint-Exupèry (2009); Las dos chelitas, de Garmendia (1984); los poemas infantiles Las lombricitas, de Nazoa (2010); El cardón y El viento, de Pushaina (2007) y el poemario (bilingüe) Olas para niños navegantes, de Ildemar (2005).

La segunda parte de la propuesta la conforma el poemario de literatura infantil bilingüe con un pequeño manual de guía para el docente, el cual es producto de esta investigación y cuya autoría se le reconoce a la investigadora participante en esta investigación, Herrera, quien es de descendencia wayuu y ha vivenciado por más de 20 años ambientes, culturas y contextos educativos de estas poblaciones indígenas. El poemario es titulado Odas a Te'pichi (Herrera, 2011), estructurado en veinte poemas y un cuento. Los poemas son: La mariposa soñadora, Pedacito de cielo, "Kai”, La niña Sue, Aromas de mi infancia, Mi burrito, Mi nube, Ensueños de jintui, "Espíritus nocturnos", "Lagunita","Zamurito azul", "El cardoncito viajero", Caballito de palo, Las hormiguitas, El volantìn, De dónde vengo, Mamá kashi, Como una mojúnai, Tuna mágica y Kaliée; y el cuento: El cuji pensador.

En las primeras sesiones prácticas, con el objetivo de alcanzar competencias comunicativas orales y escritas en los niños y niñas, se utilizó como recurso la lectura de la novela Elfie, de Lipman (2000), con su respectivo manual. Aunque esta se haya elaborado para niños de 4 a 6 años de edad, se utilizó en este nivel por considerar que estos estudiantes son mestizos, varios bilingües, pertenecen a una zona rural de muy bajos recursos, y estudian con muchas limitaciones escolares, económicas, familiares, culturales y sociales. Son escolares entre: 7, 8 y 9 años de edad y están cursando el 2do grado de primaria, muchos no saben leer (vocalizar), son muy tímidos (propio de la cultura wayuu), no se expresan espontáneamente, no socializan a través de su lenguaje. Esta realidad motivó a los investigadores para utilizar, en las sesiones posteriores, lecturas más pertinentes con su cultura y condición; el momento experimental redireccionó la investigación; por supuesto, tomando como guía los libros y propuestas de Lipman.

A continuación se presenta una muestra del trabajo de campo. (A los interesados se les invita a consultar la investigación completa). 
URL: http://www.una.ac.cr/educare

\section{Primera sesión con el Programa de FPN}

\section{Plan de acción}

Recursos: Novela Elfie

Se tomó el episodio 1

Idea principal: Los nombres

Plan de ejecución: Los nombres y su escritura

\section{Plan de discusión elaborado por los investigadores (tomado del manual de Elfie):}

1. ¿Conoces a alguien que tenga tu mismo nombre y apellido?

2. ¿Crees que en el mundo hay otras personas que tengan tu mismo nombre y apellido?

3. Crecen tus pies, ¿deberían crecer los nombres?

4. ¿Pueden tener nombres las piedras y los pájaros?

5. ¿Existen personas que no tienen nombres?

6. ¿Existe una tienda donde vendan los hombres?

\section{Ejercicios:}

Sentarse en círculos.

$>$ Cada niño dice su nombre y apellido y lo escribe.

$>$ Cada niño dice el nombre del que está a su lado.

$>$ Cada niño dice el nombre suyo, de otra persona, animal y cosa.

$>$ Escribe: Me llamo y tengo un amigo que se llama (perro, gato, peluche).

$>$ Si tuviera un pollo lo llamaría: (elefante, chivo, loro).

> Ortografía: escribir los nombres correctamente, sobre todo los que tengan las letras: B, V, S, Z, C.

$>$ Pronunciar bien los nombres de sus compañeros, conversar con los demás respetando sus nombres sin utilizar apodos.

\section{Segunda sesión con el Programa FpN}

Los nombres Elfie

Preguntas aportadas por los niños para el plan de discusión:

1. ¿Cómo es el nombre de la maestra? (Arnaldo y Andrés)

2. ¿Por qué las letras son así? (Sandro)

3. ¿Por qué es importante conocer el abecedario? (Rodolfo)

4. ¿Para qué sirven los nombres? (Yenderson)

5. ¿Para qué sirven las vocales? (Yonel)

6. ¿Para qué sirve el marcador? (José Benedicto)

\section{Ejercicios:}

- Las preguntas se colocaron en la pizarra y se fueron respondiendo.

- Luego las escribieron en su hoja diaria para el portafolio

- Ejercicios: todos escribieron en una cartulina su nombre y apellido usando mayúscula en la primera letra. 
- Cada uno dijo su nombre y apellido, el del compañero y de un animal que les gusta (mascota)

- Se escribió el abecedario y vocales en la pizarra para entender cómo se escriben los nombres y apellidos.

Observaciones:

- Todavía muy pocos respetan el turno de palabra.

- Hay poca participación.

- Cuando participan lo hacen todos a la vez.

- Muchos no conocen las letras del abecedario.

- Participaron varios que eran muy tímidos (José Benedicto, Dailybeth, Yonel)

\section{Sesiones con poemas aplicando el Programa FpN}

Poema: El cardón.

Autor: Juan Pushaina (2007)

Preguntas elaboradas por los investigadores para el plan de discusión:

1. ¿Qué quiere decir el poema?

2. ¿Ustedes conocen los cardones?

3. ¿Dónde nacen los cardones?

4. ¿Alguno de ustedes ha jugado con los cardones?

5. ¿Porque los cardones tienen púas quiere decir que son malos?

6. ¿Por qué son importantes los cardones?

Preguntas e ideas aportadas por los niños para el plan de discusión:

- "Que si yo canto el cardoncito lo atrapa" (Dijo Yenderson)

- "Cuando uno está cantando el cardoncito atrapa el viento" (José David))

- "Atrapa las cosas" (Wiliannis)

- "El cardón atrapa las canciones" (Rodolfo)

- ¿A quién de ustedes los ha puyado un cardón? (dirigida al grupo) (Yorgelis)

- "Los niños de por aquí hacemos carritos con los cardoncitos" (Enmanuel)

- "Los cardones puyan como una culebra que muerde" (sentido metafórico) (Yonel)

\section{Ejercicios:}

$>$ Sentarse en círculos

$>$ Cada niño participa pidiendo la palabra y comenta sus experiencias con esta planta.

$>$ Uso del diccionario para escribir correctamente cardón, viento, sabana y otras palabras extraídas del poema.

$>$ Elaborar un dibujo de un cardoncito con un niño teniendo un pequeño diálogo. Si fueras tú el niño, ¿qué le dirías? 
URL: http://www.una.ac.cr/educare

Poemario: Olas para niños navegantes (Ildemar, 2005)

Poema: Dormilona

\section{Preguntas elaboradas por los investigadores para el plan de discusión}

1. ¿Qué son las olas?

2. ¿Quién conoce el lago?

3. ¿Qué significa el lago para los zulianos?

4. ¿El cielo está en el cielo, y por qué el lago está en la tierra?

5. ¿Vez al lago cuando miras al cielo? ¿Por qué?

\section{Preguntas e ideas aportadas por los niños para el plan de discusión:}

- El lago está en el árbol como un pájaro (Yenderson)

- ¿Por qué existen los lagos y los ríos? (Sandro)

- "Si no existieran los lagos y los ríos se mueren los peces, y el agua sirve para beber, bañarse, lavar, ¡el agua es vida!” (Enmanuel y Rodolfo)

- "El viento sopla, el agua y las olas se hacen más altas y peligrosas" (Yenderson)

- "Por eso se forman los tsunamis que son olas muy gigantes y destruyen ciudades y todo se lo llevan; como pasó en Japón" (Jesús Andrés y Anyerbi)

- ¡Yo conozco el puente sobre el lago es maravilloso! (Enmanuel)

- "Los puentes sirven para pasar, aquí por mi casa hay un puentecito está muy viejo, da miedo pasar por él” (Erika y Wliannis)

- "El agua es importante es especial para el planeta" (Sandro)

- "Pero el agua del lago no se toma porque es salada" (José David)

- "En la laguna de Sinamaica hay un puente y una escuela dentro del agua, yo fui para allá a visitar un familiar con mi mamá" (Laure)

- "Claro, José David, se toma es el agua de río porque es dulce" (Sandro)

\section{Ejercicios:}

$>$ Sentados todos en círculos.

$>$ Los niños participan respetando el turno de palabra y comentan sus experiencias.

$>$ Un voluntario va escribiendo en la pizarra los aportes, luego copian.

> Realización de ejercicios colocando el nombre correcto a los diferentes peces y animales, colorearlos.

$>$ Escribir cinco oraciones que se refieran al cuidado y protección del lago, usando el diccionario para la ortografía.

La segunda parte de esta propuesta está constituida por un poemario de literatura infantil bilingüe (español y wayuunaiki) y su manual de discusión para el docente basado en la metodología del diálogo propuesto por Matthew Lipman con su Programa de Filosofía para Niños"; el cual nació de las entrañas de las sesiones desarrolladas con los niños con el método lipminiano; producto de la dinámica y riqueza de la investigación. 
A continuación se presenta como muestra un poema con su plan de discusión para el docente:

\section{La mariposa soñadora}

Linda mariposita de ensueño

enséñame tus secretos, cómo vuelas tan alto y te metes en mis sueños. Yo quiero ser como tú libre y emprendedora para alcanzar las estrellas y disfrutar el ahora.

Llévame a volar contigo para descubrir las riquezas que los hombres sabios construyen con firmezas.

Regálame tus alas para que el viento me guíe;

y así alcanzar el corazón de mi madre

¡qué desde el cielo me sonríe!

\section{Plan de discusión para el poema: "La mariposa soñadora"}

1. ¿Les gustó el poema? ¿Qué es un poema?

2. ¿Por qué se llama así?

3. ¿Quién ha jugado con las mariposas?

4. ¿Qué significa ensueño?

5. ¿Quién quiere contar un sueño?

6. ¿Quién ha guardado un secreto?

7. ¿Qué significa volar? ¿Por qué solo vuelan los pájaros?

8. ¿Por qué las personas no vuelan?

9. ¿Se pueden alcanzar las estrellas? ¿Por qué?

10. ¿Qué significan los dos últimos versos? ¿Qué son versos?

11. ¿En qué se relaciona el sueño con la realidad?

12. ¿Los sueños se convierten en realidad?, ¿cómo y por qué?

\section{Ejercicios:}

1. Todos los niños se sientan en círculo van participando en orden, respetando el turno de palabra, según las preguntas que vaya formulando el docente y las que vayan surgiendo.

2. Un voluntario las escribe en la pizarra y democráticamente escogen las más interesantes.

3. JUEGO: varios niños simulan ser una niña que habla con la mariposa siguiendo la musicalidad del poema.

4. Los voluntarios que deseen participar contarán algún sueño y qué significado tiene para ellos.

5. Pasarán a la pizarra a escribir algunas palabras en español y en wayuunaiki 
URL: http://www.una.ac.cr/educare

\section{Conclusiones}

A manera de conclusión se afirma que el Programa de Filosofía para Niños es eficaz para el desarrollo de la competencia comunicativa de los estudiantes de educación primaria, como lo demostraron los resultados de esta investigación.

Los aportes obtenidos en esta investigación permitieron a los niños y niñas descubrir que la escuela es un ambiente enriquecedor, donde la lectura debe ser una actividad de disfrute y recreación. Es la fuente donde fluye su pensamiento crítico-reflexivo. Se obtiene el valor agregado de darle a la lectura la importancia que tiene como medio esencial para su formación integral y para alcanzar el éxito en todos los contextos de su vida cotidiana.

Asimismo, a través de la lectura de obras literarias contextualizadas y la aplicación de la metodología del diálogo filosófico en una comunidad de investigación (Lipman), donde se les brinde un ambiente de armonía, respeto y valor por sus ideas; los niños y niñas se identificaron con dichas lecturas, se sintieron motivados para expresarse libremente; por ello desarrollaron sus competencias comunicativas de forma oral y escrita adecuándolas a su situaciones de la vida diaria.

Otro aporte relevante fue que el Programa FpN ofrece la capacitación para el docente en cuanto a novedosas metodologías y recursos para alcanzar un aprendizaje significativo, le brinda herramientas muy efectivas para aprovechar la interdisciplinariedad; ya que el programa es muy completo puesto que la Filosofía es la disciplina que prepara a los estudiantes para pensar en las disciplinas: Lenguaje, Matemáticas, Ciencias, Educación Física y Ciencias Sociales a través de: leer, escribir, hablar, escuchar.

Por otro lado, esta experiencia de investigación sobre el Programa FpN y el trabajo de campo con el grupo experimental motivó a uno de los investigadores, Yamelis Herrera, para la creación de un libro de literatura infantil contextualizada, como recurso didáctico para facilitar el desarrollo del pensamiento crítico-reflexivo y alcanzar la competencia comunicativa en los niños desde temprana edad.

\section{Referencias}

Accorinti, S. (septiembre, 2002). Matthew Lipman y Paulo Freire: Conceptos para la libertad. Utopía y praxis Latinoamericana, 7(18), 35-56.

Arias, F. (2006). El proyecto de investigación. Introducción a la metodología científica (5ª ed.). Caracas, Venezuela: Editorial Episteme.

Bruzual, R. (2002). Propuesta comunicativa para la enseñanza de la lengua materna. Maracaibo, Venezuela: Universidad del Zulia. Ediciones Astro Data.S.A Maracaibo, Venezuela. (Tesis publicada)

Carmona, (2007). Formación del docente y filosofía para niños y niñas. (Tesis Doctoral). Universidad del Zulia. Zulia, Venezuela. 
Canale, M. (1983). From Communicative Competence to Communicative Language Pedagogy. [De la competencia comunicativa a la Pedagogía del lenguaje comunicativo]. En J. Richards y J. Schdmit (Eds.) Language and Communication (pp. 2-27). London: Longman.

Canale, M. y Swain, M. (1980). Theoretical Bases of Communicative Approches to Second Language Teaching and Testing [Bases teóricas del enfoque comunicativo para la enseñanza y prueba del un segundo idioma]. Applied Linguistics, 1(1), 1-47. Doi. 10.1093/applin/I.1.1

Castillo, J. A. (junio, 2008). El desarrollo de la expresión oral a través del taller como estrategia didáctica globalizadora. Sapiens, 9(1), 179-203.

Chávez, N. (2007). Introducción a la investigación educativa. Maracaibo. Estado Zulia: Ars Grafic.

De Saint-Exupèry, A. (2009). El Principito. Caracas, Venezuela: Ediciones El Trébol siglo XXI.

Franco, A. (1999). Curso de lengua española (reimpresión de la $3^{\text {a }}$ ed.). Mérida, Venezuela: Ediluz.

Franco, A. (2007). Gramática comunicativa. Mérida, Venezuela: Ediluz.

Garmendia, J. (1984). Las dos chelitas. En G. Meneses (Comp.). Antología del cuento venezolano (pp. 81-83). Caracas: Monte Ávila.

Herrera, Y. (2011). Odas a Te’pichi. (Inédito). Maracaibo. Venezuela.

Hymes, D. (1996). Acerca de la competencia comunicativa. Forma y función, 9, 13-37.

Hymes, D. (1971). Competence and Performance in Linguistic Theory [Competencia y el rendimiento en la teoría lingüística]. En R. Huxley y E. Ingram (eds.). Languaje Acquisition: Models and Methods [Adquisicion del lenguaje: Modelos y métodos] (pp. 3-23). New York: Academic Press.

Ildemar, C. (2005). Olas para niños navegantes. Maracaibo, Venezuela: Ediluz.

Lipman, M. (1998). Pensamiento complejo y educación. Madrid: Ediciones La Torre.

Lipman, M. (2000). Elfie. Madrid: Ediciones de La Torre.

Lipman, M. y Gazard, A. (2001). Poner nuestros pensamientos en orden. Manual para acompañar a Elfie. (Proyecto didáctico Quirón). (Trad. P. Pedraza Moreno en colaboración de F. García Moriyón). Madrid. España: Ediciones de La Torre.

Lipman, M., Sharp, A. M. y Oscanyan, F. S. (2002). La Filosofía en el Aula. Madrid: Ediciones de la Torre. 
Lomas, C. (1999). Cómo enseñar a hacer cosas con las palabras. Teoría y práctica de la educación lingüística. Barcelona: Editorial Paidós.

Martínez, M. C. (2001). Análisis del discurso y práctica pedagógica. Una propuesta para leer, escribir y aprender mejor ( $3^{\mathrm{a}}$ ed.). Rosario, Santa Fe, Argentina: Homo Sapiens.

Molero, L. (1998). Un modelo lingüístico para la planificación de la enseñanza de la lengua materna. Teoría y práctica. Zulia, Maracaibo: Fundacite.

Nazoa, A. (2010). Las lombricitas. Colombia: Playco Editores.

Ocampo, J. (2008). Paulo Freire y la pedagogía del oprimido. Revista Historia de la Educación Latinoamericana, 10, 57-72.

Prado, J. (2004a). Didáctica de la Lengua y la Literatura: Maestro de educación primaria (Proyecto docente). España: Universidad de Huelva.

Prado, J. (2004b). Didáctica de la lengua y la literatura para educar en el siglo XXI. Madrid: La muralla.

Pushaina, J. (2007). Poemas inéditos. Maracaibo, Venezuela.Saussure, F. (1983). Curso de lingüística general. Madrid: Alianza Editorial. Manuscrito inédito.

Serrón, S. (2002). El enfoque comunicativo y sus implicaciones. Una visión desde la enseñanza de la lengua materna en un marco democrático. Letras, 62, 225-260.

Tellez, A. y Bruzual, R. (diciembre, 2005). Diagnosis del proceso de compresión lectora en la segunda etapa de educacion básica. Paradigma, 26(2), 77-98. 